\title{
Reanalysis of the Gas-cooled fast reactor experiments at the zero power facility Proteus - Spectral indices
}

\author{
G. Perret ${ }^{1}$, R. M. Pattupara ${ }^{1}$, G. Girardin ${ }^{2}$, and R. Chawla ${ }^{1,2}$ \\ ${ }^{1}$ Paul Scherrer Institute, NES Department, 5232 Villigen, Switzerland \\ ${ }^{2}$ Ecole Polytechnique Fédérale de Lausanne, 1015 Lausanne, Switzerland
}

\begin{abstract}
PROTEUS is a zero power reactor at the Paul Scherrer Institute which has been employed during the 1970's to study experimentally the physics of the gas-cooled fast reactor. Reaction rate distributions, flux spectrum and reactivity effects have been measured in several configurations featuring $\mathrm{PuO}_{2} / \mathrm{UO}_{2}$ fuel, absorbers, large iron shields, and thorium oxide and thorium metal fuel either distributed quasihomogeneously in the reference $\mathrm{PuO}_{2} / \mathrm{UO}_{2}$ lattice or introduced in the form of radial and axial blanket zones. This papers focus on the spectral indices - including fission and capture in ${ }^{232} \mathrm{Th}$ and ${ }^{237} \mathrm{~Np}$ - measured in the reference $\mathrm{PuO}_{2} / \mathrm{UO}_{2}$ lattices and their predictions with an MCNPX model specially developed for the PROTEUS-GCFR core. Predictions were obtained with JEFF-3.1 and -3.11, ENDF/B-VII.0 and VII.1, and JENDL-3.3 and -4.0. A general good agreement was demonstrated. The ratio of ${ }^{232} \mathrm{Th}$ fission to ${ }^{239} \mathrm{Pu}$ fission, however, was under-predicted by $8.7 \pm 2.1 \%$ and $6.5 \pm 2.1 \%$ using ENDF/B-VII.0 and VII.1, respectively. Finally, the capture rates in ${ }^{237} \mathrm{~Np}$ tended to be underpredicted by the JEFF and JENDL libraries, although the new cross section in JEFF-3.1.1 slightly improved the ${ }^{237} \mathrm{~Np}$ capture to ${ }^{239} \mathrm{Pu}$ fission results $(3.4 \pm 2.4 \%)$.
\end{abstract}

\section{Introduction}

The Gas-cooled Fast Reactor (GFR) concept has been proposed by Generation-IV (GEN-IV) initiative to develop safe, sustainable, reliable, proliferation-resistant and economic nuclear energy systems [1]. In this context, the neutronic and thermal-hydraulic properties of the GFR have been recently analysed within the Nuclear Energy and Safety division of the Paul Scherrer Institute (PSI), using the in-house code system FAST [2].

Interest in gas-cooled fast reactors (GCFRs), however, dated back to the 1970's and, at the time, the neutronics of early GCFR designs was investigated experimentally in the PROTEUS zero power reactor at PSI. The experimental programme comprised a large set of configurations to validate lattice and core calculation methods, and associated nuclear data libraries. Most of the experiments were related to $\mathrm{PuO}_{2} / \mathrm{UO}_{2}$ fuelled GCFRs, i.e. to the commonly applied ${ }^{238} \mathrm{U} /{ }^{239} \mathrm{Pu}$ fuel cycle. However, several core configurations were dedicated to nuclear data validation for the alternative ${ }^{232} \mathrm{Th} /{ }^{233} \mathrm{U}$ fuel cycle [3].

Experimental results were compared in the 1970's with 1D and 2D deterministic predictions obtained using the SN-1D/DIFF-1D and DIFF-2D code systems, in conjunction with ENDF/B-IV and FGL5 cross-sections prepared by the GGC-4 and MURLAB cell codes. In the frame of a recent Master's thesis [4], a new 3D MCNPX model of the full GCFR-PROTEUS reactor has been

This is an Open Access article distributed under the terms of the Creative Commons Attribution License 2.0, which permits unrestricted use, distribution, and reproduction in any medium, provided the original work is properly cited. 
developed. As a first step, the model is used in this paper to predict spectral indices measured in the centre of the reactor test zone and to validate the cross sections of the traditional plutonium and uranium isotopes and that for ${ }^{237} \mathrm{~Np}$ and ${ }^{232} \mathrm{Th}$ capture and fission, ${ }^{233} \mathrm{U}$ fission, and $(\mathrm{n}, 2 \mathrm{n})$ reaction in ${ }^{232} \mathrm{Th}$. Calculated predictions are based on the modern continuous energy libraries JEFF-3.1 and 3.1.1 (J31, J311), ENDF/B-VII.0 and VII.1 (B70, B71), and JENDL-3.3 and -4.0 (JN33, JN40).

The following section reviews the characteristics of the experimental configuration of the GCFRPROTEUS programme considered in this paper. The calculation models are briefly discussed in Section 3, while the comparison of the calculated spectral indices with measured values is presented in Section 4. The final section provides the conclusions.

\section{Experiments}

PROTEUS is a zero power reactor featuring a cylindrical central cavity that is driven critical by a surrounding graphite region fuelled with $5 \mathrm{w} \% \mathrm{UO}_{2}$ fuel pins. In the GCFR experiments, the central cavity was loaded with a $\mathrm{D}_{2} \mathrm{O}$ zone fuelled with $5 \mathrm{w} \% \mathrm{UO}_{2}$ fuel pins, a buffer region containing metallic natural uranium rods in air and a central test zone filled with a GCFR-representative fuel lattice. This multi-zone arrangement has the advantage of reducing, by a factor of $\sim 10$, the amount of plutonium required to obtain a critical mock-up representative of the fast reactor under study.

In this paper we focus on the GCFR reference configuration which featured in the central test zone a regular hexagonal lattice $\left(1 \mathrm{~cm}\right.$ pitch) of $\mathrm{PuO}_{2} / \mathrm{UO}_{2}$ fuel rods. The fuel contained $15 \mathrm{w} \% \mathrm{Pu}$ of which $80 \%$ was fissile - and was clad in stainless steel. The fuel pellets had a diameter of $6.7 \mathrm{~mm}$ and a density of $10.6 \mathrm{~g} / \mathrm{cm}^{3}$ and provided an active length of $\sim 0.83 \mathrm{~m}$ sandwiched between a top and a bottom blanket of depleted uranium $\left(0.42 \mathrm{w} \%{ }^{235} \mathrm{U}, 10.5 \mathrm{~g} / \mathrm{cm}^{3}\right)$ to make up a total fuel length of $1.4 \mathrm{~m}$.

Reaction rate ratios were measured using metal foils $\left({ }^{238} \mathrm{U},{ }^{232} \mathrm{Th}\right)$, aluminium-alloyed fission foils $\left({ }^{239} \mathrm{Pu},{ }^{235} \mathrm{U},{ }^{233} \mathrm{U}\right)$ and thin deposits on aluminium backing $\left({ }^{237} \mathrm{~Np}\right)$. Because the number of heavy atoms in the aluminium-alloyed foils could not be determined accurately, spectral indices relative to fissions in ${ }^{239} \mathrm{Pu}$ required the use of additional calibrated ${ }^{239} \mathrm{Pu}$ deposits in back-to-back fission chambers. More details on the measurement technique can be found in [5]. Using $\mathrm{C}$ and $\mathrm{F}$ as abbreviations for capture and fission, and 2, 3, 5, 7, 8 and 9 for ${ }^{232} \mathrm{Th},{ }^{233} \mathrm{U},{ }^{235} \mathrm{U},{ }^{237} \mathrm{~Np},{ }^{238} \mathrm{U}$ and ${ }^{239} \mathrm{Pu}$, the total uncertainty on the measured spectral indices were $1.1-1.3 \%$ for C8/F9, F8/F9, F5/F9, F3/F9 and $\mathrm{C} 2 / \mathrm{F} 9,1.8-2 \%$ for F7/F9 and F2/F9, and 2.3-2.5\% for C7/F9 and (n,2n)2/C2.

\section{Calculations}

Cell and 3D full core model of the GFCR reference core configurations were developed with the MCNPX code. Top and side cut-away views of the full core model are shown in Fig. 1. The cell model has been used in conjunction with the $3 \mathrm{D}$ model to demonstrate that the spectrum in the centre of the PROTEUS test zone - where the measurements have been performed - is similar to that of an infinite lattice. Both flux and spectral indices have been compared and shown to be adequately similar [5].

In the full core model, the flux and reaction rates of interest were tallied in the central pins of the test zone in which the spectrum is as close as possible to that in a single zone reactor. Axially, the tallies were limited to $10 \mathrm{~cm}$ to prevent any spectral distortion due to the depleted uranium blankets. Calculations were run with ENDF/B-VII.0 and VII.1 (B70, B71), JEFF-3.1 and -3.1.1 (J31, J311) and JENDL-3.3 and -4.0 (JN33, JN40). The differences in keff values were less than $200 \mathrm{pcm}$. Calculations were run with 250 to 500 million neutrons depending on the nuclear data library. Monte Carlo uncertainties on the spectral indices were negligible $(\sigma<0.5 \%)$ as compared to the measurement uncertainty. The only exception was for the spectral index involving $(n, 2 n)$ reactions in thorium $(\sigma \sim 3 \%)$, because $(n, 2 n)$ reactions in ${ }^{232}$ Th have a high threshold of about $6.5 \mathrm{MeV}$. 

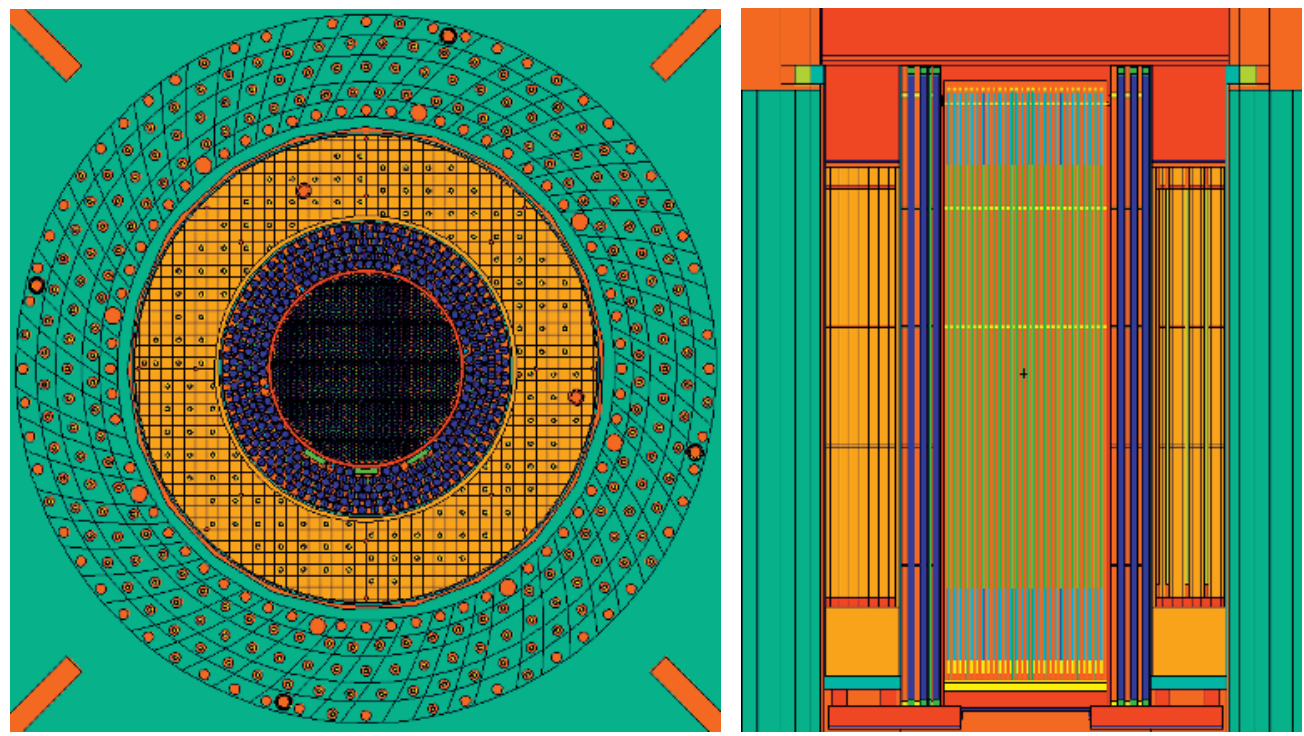

Fig. 1. Top and side cut away view of the full core MCNPX model.

In addition a reference flux calculation using the B71 library has been performed with the detailed SAND-II energy group structure and reaction rates has been tallied in the core centre using this flux and cross sections from different libraries. This allowed singling out the impact of the different library cross section for a typical GCFR spectrum.

\section{Results and Discussions}

\subsection{GCFR reaction rates calculated with different libraries}

The reaction rates obtained with different cross sections and the flux calculated with the MCNPX full core model and the B71 library is shown in Tab. 1. The reaction rates are normalised to those obtained with B71. Because the same flux calculated with MCNPX is used, no Monte Carlo uncertainty has to be considered.

Table 1. Reaction rate predictions obtained for a reference GCFR flux calculated with the full core MCNPX model and cross sections from different libraries.

\begin{tabular}{cccccc}
\hline Reaction & B70/B71 & J31/B71 & J311/B71 & JN33/B71 & JN40/B71 \\
\hline F5 & 1.000 & 1.002 & 1.002 & 0.999 & 0.990 \\
F8 & 1.000 & 0.996 & 0.996 & 1.004 & 0.999 \\
F9 & 1.000 & 0.997 & 0.997 & 1.002 & 1.005 \\
(n,2n)2 & 1.002 & 1.020 & 1.020 & 0.851 & 0.987 \\
F2 & 0.976 & 1.046 & 1.046 & 1.042 & 1.059 \\
C8 & 0.999 & 1.003 & 1.003 & 1.028 & 1.002 \\
C2 & 0.994 & 0.976 & 0.976 & 0.916 & 0.983 \\
F3 & 1.000 & 1.005 & 1.005 & 1.005 & 1.006 \\
F7 & 1.000 & 1.001 & 0.963 & 1.001 & 0.992 \\
C7 & 1.002 & 0.960 & 0.972 & 0.960 & 0.974 \\
\hline
\end{tabular}


As expected, all reactions in ${ }^{235} \mathrm{U},{ }^{238} \mathrm{U},{ }^{233} \mathrm{U}$ and ${ }^{239} \mathrm{Pu}$ are consistent between libraries - except for capture in ${ }^{238} \mathrm{U}$ in $\mathrm{JN} 33$ whose cross section have been subsequently modified below $20 \mathrm{keV}$ in J40 [6]. It confirms that using F9 as the denominator in the measured spectral indices will not induced additional bias when comparing the libraries in the following section. The update to B71 from B70 does not change significantly the reaction rates of interest with the exception of fission in ${ }^{232} \mathrm{Th}$ (F2). A detailed energy distribution of F2 shows that the total increase of $2.4 \%$ mainly comes from neutrons with energies between 1.5 and $4 \mathrm{MeV}$. Capture in ${ }^{232} \mathrm{Th}$ is shown to be consistent for $\mathrm{J} 31, \mathrm{~J} 311$ and $\mathrm{J} 40$ and about 2\% less than for the B70 and B71 libraries. Finally, the new value for the ${ }^{237} \mathrm{~Np}$ fission cross section in J311 [7] is shown to underestimate the values obtained with all other libraries by about $4 \%$, and the ${ }^{237} \mathrm{~Np}$ capture values obtained with B70 and B71 are 3 to $4 \%$ higher than for the other libraries.

\subsection{Spectral indices predictions compared to experimental values}

Predictions of the spectral indices obtained with the different nuclear libraries shown above are compared to experimental values in Tab. 2. Relative uncertainties are given at $1 \sigma$ and account for Monte Carlo and experimental uncertainties. Discrepancies between 2 and $3 \sigma$ and above $3 \sigma$ are printed in italic and bold, respectively.

Table 2. Calculation-to-experiment ratios for spectral indices in the reference lattice for different nuclear data library.

\begin{tabular}{ccccccc}
\hline SI & B70 & B71 & J31 & J311 & JN33 & JN40 \\
\hline C8/F9 & $0.995(1.2 \%)$ & $0.997(1.2 \%)$ & $0.993(1.2 \%)$ & $0.991(1.2 \%)$ & $0.993(1.2 \%)$ & $0.981(1.2 \%)$ \\
F8/F9 & $1.009(1.4 \%)$ & $1.009(1.4 \%)$ & $0.992(1.4 \%)$ & $0.992(1.4 \%)$ & $1.032(1.4 \%)$ & $1.008(1.4 \%)$ \\
F5/F9 & $1.012(1.5 \%)$ & $1.013(1.5 \%)$ & $1.012(1.5 \%)$ & $1.011(1.5 \%)$ & $1.009(1.5 \%)$ & $0.991(1.5 \%)$ \\
C2/F9 & $1.015(1.4 \%)$ & $1.032(1.5 \%)$ & $0.985(1.5 \%)$ & $0.994(1.6 \%)$ & $\mathbf{0 . 9 3 1 ( 1 . 6 \% )}$ & $0.985(1.5 \%)$ \\
F2/F9 & $\mathbf{0 . 9 1 3 ( 2 . 1 \% )}$ & $\mathbf{0 . 9 3 5 ( 2 . 1 \% )}$ & $0.965(2.1 \%)$ & $0.965(2.1 \%)$ & $0.996(2.1 \%)$ & $0.991(2.1 \%)$ \\
F3/F9 & $0.987(1.4 \%)$ & $0.986(1.4 \%)$ & $0.992(1.4 \%)$ & $0.995(1.4 \%)$ & $0.990(1.4 \%)$ & $0.983(1.4 \%)$ \\
$(\mathrm{n}, 2 \mathrm{n}) 2 / \mathrm{C} 2$ & $\mathbf{1 . 0 8 4 ( 2 . 9 \% )}$ & $1.054(2.9 \%)$ & $\mathbf{1 . 1 1 2 ( 3 . 2 \% )}$ & $1.051(3.8 \%)$ & $1.026(3.8 \%)$ & $\mathbf{1 . 1 2 6 ( 3 \% )}$ \\
C7/F9 & $1.003(2.4 \%)$ & $1.000(2.4 \%)$ & $0.951(2.4 \%)$ & $0.966(2.4 \%)$ & $0.953(2.4 \%)$ & $0.960(2.4 \%)$ \\
F7/F9 & $1.003(1.9 \%)$ & $1.003(1.9 \%)$ & $1.006(1.9 \%)$ & $0.969(1.9 \%)$ & $1.006(1.9 \%)$ & $1.002(1.9 \%)$ \\
\hline
\end{tabular}

Fission in ${ }^{235} \mathrm{U}$ and ${ }^{233} \mathrm{U}$, as compared to fission in ${ }^{239} \mathrm{Pu}$, is well predicted with all libraries. Fissions in ${ }^{238} \mathrm{U},{ }^{237} \mathrm{~Np}$ and ${ }^{232} \mathrm{Th}$ are threshold reactions (0.5 to $1 \mathrm{MeV}$ thresholds) and are well predicted by the majority of nuclear libraries. Fission in ${ }^{238} \mathrm{U}$ is slightly overestimated with JN33 (as shown in Section 4.1) and fission in ${ }^{237} \mathrm{~Np}$ is only slightly underpredicted in $\mathrm{J} 311$ due to the new cross section [7]. Fission in ${ }^{232} \mathrm{Th}$ is, however, overpredicted by $8.7 \pm 2.1 \%$ and $6.4 \pm 2.1 \%$ by B70 and $\mathrm{B} 71$. As can be seen from Tab. 1, the underestimation is mainly due to the new ${ }^{232} \mathrm{Th}$ fission crosssections in B70 and B71 which was re-evaluated using the latest measurements performed at the n_TOF and GELINA facilities [8].

Capture in ${ }^{238} \mathrm{U}$ is well predicted by all libraries. Captures in ${ }^{232} \mathrm{Th}$ is also well predicted except for JN33. ${ }^{237} \mathrm{~Np}$ captures are slightly underestimated with JN33 and J31. The new evaluation of the capture cross section of ${ }^{237} \mathrm{~Np}$ in $\mathrm{J} 311$ is mainly in the thermal and resonance range. In the fast GCFR-PROTEUS spectrum, the new cross section slightly improves the prediction by about $1 \%$. Note that the uncertainties on the measured $\mathrm{C} 2 / \mathrm{F} 9$ and $\mathrm{C} 7 / \mathrm{F} 9$ spectral indices are $1.3 \%$ and $2.3 \%$, respectively. Longer MCNPX runs would therefore not change the picture significantly.

For the $(n, 2 n) 2 / C 2$ spectral index, the uncertainty on the calculations is not negligible as compared to the uncertainty on the experimental value $(2.5 \%)$. (n,2n)2/C2 spectral index is significantly overestimated $(\sim 8-13 \pm 3 \%)$ using B70, J31 or JN40. On the contrary, values for B71, $\mathrm{J} 311$ and JN33 are in reasonable agreement with the experiments. (n,2n) and capture cross sections for ${ }^{232} \mathrm{Th}$ are however the same in $\mathrm{J} 31$ and $\mathrm{J} 311$, and the corresponding reaction rates in the GCFR 
spectrum are almost identical for B70 and B71 (see Tab. 1). Differences in the flux above $6.5 \mathrm{MeV}$ together with the inherent fluctuation of the Monte Carlo process should thus explain the difference.

\section{Conclusions}

Early designs of the gas cooled fast reactor were investigated experimentally in the zero power facility PROTEUS during the 1970s. Recently, measured results has started to be re-interpreted with the help of a full core Monte Carlo MCNPX model and modern libraries such as JEFF-3.1 and 3.1.1, ENDF/B-VII.0 and -VII.1, and JENDL-3.3 and -4.0. This paper presented the validation of the different libraries using spectral indices, for traditional uranium and plutonium but also ${ }^{232} \mathrm{Th}$ and ${ }^{237} \mathrm{~Np}$, measured in the centre of the reference $\mathrm{PuO}_{2} / \mathrm{UO}_{2}$ lattice.

A good agreement has been generally demonstrated for the investigated nuclear data libraries. Among the remaining discrepancies, fission in ${ }^{232} \mathrm{Th}$ has been shown to be significantly underestimated using ENDF/B-VII.0 and -VII.1 (8.7 and $6.4 \pm 2.1 \%$, respectively), and (n,2n) reactions in ${ }^{232} \mathrm{Th}$ have been hard to predict with a good accuracy with the Monte Carlo code. Finally, the recent adjustment of the ${ }^{237} \mathrm{~Np}$ capture cross section in JEFF-3.1.1 (as compared to JEFF3.1 ) has slightly improved the agreement with the measured ${ }^{237} \mathrm{~Np}$ capture-to- ${ }^{239} \mathrm{Pu}$ fission rate ratio ( 0.951 to $0.966 \pm 2.4 \%$ ), whereas the change of the ${ }^{237} \mathrm{~Np}$ fission cross section has worsen the agreement with the measured ${ }^{237} \mathrm{~Np}$ fission-to- ${ }^{239} \mathrm{Pu}$ fission rate ratio $(1.006$ to $0.969 \pm 1.9 \%)$.

This work demonstrated that the past GCFR experiments at PROTEUS can still help to validate modern cross section libraries. Such work could be extended to spectral indices measured in other configurations of the experimental programme, which featured thorium oxide and metallic fuel inserted quasi homogeneously or in the form of axial and radial blanket zones, but also to other measured quantities such as flux spectra and reaction rate distributions.

\section{References}

1. R. Stainsby et al., Science and Technology of Nuclear Installations, 241, pp. 3481-3489 (2009).

2. K. Mikityuk, Proceeding of ICONE17, Brussel, Belgium, July 12-16 (2009).

3. R. Richmond, Eidg. Institut für Reaktorforshung - EIR Report 478.

4. R. M. Pattupara, EPFL Master Thesis (2011).

5. G. Perret et al., Proceeding of Physor 2012, Knoxville, Tennessee, USA, April 15-20 (2012).

6. K. Shibata et al., Journal of Nuclear Science and Technology, 48, pp. 1-30 (2011).

7. A. Santamarina et al., JEFF Report 22, OECD, NEA No. 6807 (2009).

8. R. Capote et al., IAEA Report 1435 (2010). 